\title{
Distributed $H_{2} / H_{\infty}$ Consensus Control for Multi-agent Systems with Directed Graph and Actuator Uncertainty
}

\section{Yichao Ao and Yingmin Jia}

School of Automation Sciences \& Electrical Engineering, BeiHang University, XueYuan Road No.37, HaiDian District, BeiJing, China

\section{Abstract}

In this work, single-integrator multi-agent directed networks with energy bounded disturbances and Guassian white noises are considered in term of $\mathrm{H}_{2}$ and $\mathrm{H}_{\infty}$ performance. Meanwhile actuator uncertainty is also the concern of $H_{\infty}$ performance. Distributed control law of undetermined parameters is proposed. By using $H_{2} / H_{\infty}$ theory and linear algebra, the problem is transformed into a non-convex optimization problem on diagonal matrices. Numerical iterative algorithms with consideration on both feasibility and conservatism are presented.

\section{Publication History:}

Received: March 01, 2018

Accepted: April 18, 2018

Published: April 20, 2018

\section{Keywords:}

Multi-agent systems, Consensus, $\mathrm{H}_{2} / \mathrm{H}_{\infty}$ control, Non-convex optimization, Actuator uncertainty, Iterative approach

\section{Introduction}

In last two decades, consensus control of multi-agent systems has been researched deeply and extensively, for its broad range of applications in several areas, such as: robotic teams cooperation, sensor networks, air vehicles formation flying, social networks and so on [20-24]. And it has attracted the attention from several fields including but not limited to engineering, biology, control theory and computer science.

For consensus problems, abundant achievements for first-order agents have been acquired by previous researchers [1-4]. The study of the multi-agent systems was from undirected graph to directed graph, from fixed communication topology to switched topology, and with time-delay or not. However, under real circumstances, the transmission errors, communication obstacles, actuator bias and other disturbances do exist. From now on, several researchers had investigated the disturbance rejection performance of multi-agent systems in different perspectives. P. Lin [5,6] firstly introduced $H_{\infty}$ control theory to multi-agent systems. In his work, An orthogonal transformation was utilized to guarantee the validity of $H_{\infty}$ control theory. And Y. Liu [7] investigated multi-agent systems of high-order integrator and general linear time-invariant dynamics by $H_{\infty}$ criterion. Consensus protocols involving a coefficient were raised by $\mathrm{Z}$. Li and $\mathrm{Y}$. Zhao $[9,10]$, and the $H_{\infty}$ performance for both state feedback controlled systems and output feedback controlled systems was investigated by introducing $H_{\infty}$ performance region of the coefficient. It is worthy mentioning that the authors in $[9,29]$ addressed $H_{\infty}$ and $H_{2}$ consensus problems of linear multi-agent systems and proposed algorithms to satisfy the $H_{\infty}$ and $H_{2}$ conditions separately. Y. Cui [8] employed $L_{2}-L_{\infty}$ theory to solve the peak bounded consensus problems of high-order multi-agent systems with external disturbances and parameter perturbations. In other perspectives, stochastic theory and probability limit theory were introduced to consensus problems of multi-agent systems $[11,12]$, in both continuous-time and discretetime case. V. Gupta [13] and Y. Jia [15] considered the LQG problems of networks of dynamical agents. When the systems are disturbed by Gaussian noises, the designs were synthesized under $\mathrm{H}_{2}$ criteria for robust $[25,26,31]$. On another aspect of $H_{2}$ control, the recent paper [30] studied guaranteed cost problem for multi-agent systems with actuator faults and uncertainty.
In last century, numerous results were obtained in $\mathrm{H}_{2} / \mathrm{H}_{\infty}$ control. DS. Bernstein [14] proposed a LQG performance approach with an $H_{\infty}$ performance bound based on the solutions of three coupled Riccati equations. PP Khargonekar [16] simplified it to a convex suboptimization problem. D. Arzelier [15] gave a sufficient condition in the form of bilinear matrix inequalities (BMIs) to reduce the conservatism, and solved this non-convex problem by a numerical iterative algorithm. C. Scherer [27] considered the more universal case where the channels of the disturbance inputs and controlled outputs are different, when different performance indexes are concerned. However, $H_{2}$ performance and $H_{\infty}$ performance have never been considered together in multi-agent systems.

In this paper, consensus problems of single-integrator multi-agent systems with fixed directed interaction topology will be studied. We address the consensus performance subject to external $L_{2}$ disturbances and Guassian white noises by combining $H_{\infty}$ theory and $H_{2}$ theory. Our objective is to search the linear feedback controller, based on relative states of its neighbors, to optimize the $H_{2}$ performance of the multiagent systems, under two constraints. One is that the multi-agent systems reach asymptotically consensus with the absence of external signals, and the other is the constraint of $H_{\infty}$ performance against both energy bounded disturbances and actuator uncertainties. Firstly, we will present the algorithms to design the satisfactory controller, rather than proposing a theorem to verify the protocol is valid or not. Secondly, different from other work, the feedback gain matrices of the multi-agent systems are Laplacian matrices with a fixed graphical structure constraint, which has more complicated relationship between the entries. We transform it to an optimization problem in a set of diagonal matrix. To measure the consensus $\mathrm{H}_{2}$ performance, a novel output function is defined, which is linked to the LQR problem of linear-consensus. After a procedure of model transformation

"Corresponding Author: Prof. Yingmin Jia, School of Automation Sciences District, BeiJing, China, Tel: +010-82-338683; E-mail: ymjia@buaa.edu.cn

Citation: Ao Y, Jia Y (2018) Distributed $H_{2} / H_{\infty}$ Consensus Control for Multi-agent Systems with Directed Graph and Actuator Uncertainty. Int J Comput Softw Eng 3: 133. doi: https://doi.org/10.15344/2456-4451/2018/133

Copyright: (c) 2018 Ao et al. This is an open-access article distributed under the terms of the Creative Commons Attribution License, which permits unrestricted use, distribution, and reproduction in any medium, provided the original author and source are credited. \& Electrical Engineering, BeiHang University, XueYuan Road No.37, HaiDian 
Citation: Ao Y, Jia Y (2018) Distributed $\mathrm{H}_{2} / \mathrm{H}_{\infty}$ Consensus Control for Multi-agent Systems with Directed Graph and Actuator Uncertainty. Int J Comput Softw Eng 3: 133. doi: https://doi.org/10.15344/2456-4451/2018/133

Page 2 of 9

to guarantee the validity of $H_{\infty}$ theory, an original elimination lemma is derived. By using this lemma, the problem is simplified to a numerically solvable BMI constrained optimization problem on diagonal matrices. Finally, a previously proposed iterative method is used to solve the BMI optimizaiton problem.

The paper is organized as follows. In section 2, some basic frameworks of graph theory and $H_{2} / H_{\infty}$ control are introduced. $H_{2} /$ $H_{\infty}$ consensus problem for first-order directed network is described in section 3. Section 4 gives the main results and the approaches to solving the $H_{2} / H_{\infty}$ consensus problem. In section 5 , numerical simulations are presented to verify our results. Finally, conclusions are drawn in section 6 .

\section{Notations}

In this paper, $I_{n}$ represents the identity matrix with $n$ dimensions and $1_{n}$ represents $n$-dimension column vector $[1,1, \ldots, 1]^{\mathrm{T}}$. Denote $\operatorname{sym}(\mathrm{A})=\mathrm{A}+\mathrm{A}^{\mathrm{T}}$, for a square matrix $\mathrm{A}$. And $\operatorname{diag}\left\{m_{1}, m_{2}, \ldots, m_{n}\right\}$ is the diagonal matrix whose diagonal entries are given by $m_{1}, m_{2}, \ldots, m_{n}$. For a $n$-order digraph, $L_{n}$ denotes the set of all $n$-dimensional Laplacian matrices of the graph. And for a digraph with $m$ edges, $D_{m}$ denotes the set of all $m$-dimensional positive definite diagonal matrices. $L_{2}[0, \infty)$ represents the space of square integrable vector functions over $[0, \infty)$.

\section{Preliminaries}

\section{Graph theory}

Let $G=(v, \varepsilon, A)$ be a weighted directed graph of $\mathrm{n}$ orders, which consists of a set of nodes $V=\left\{v_{1}, v_{2}, \ldots, v_{n}\right\}$, a set of edges $\varepsilon \subseteq V \times V$, And a weighted adjacency matrix $A=\left[a_{i j}\right]$. Node pair $\left(v_{i}, v_{i}\right) \in \varepsilon$ implies that there is a formation flow from $v_{j}$ to $v_{i}$, where $v_{i}$ and $v_{j}$ call the tail of the edge and the head of the edge, respectively. The neighbor of vi is defined by $N_{i}=\left\{v_{j} \in V:\left(v_{i}, v_{j}\right) \in \varepsilon\right\}$. And the elements of adjacency matrix A are nonnegative, in which $\mathrm{a}_{\mathrm{ij}}>0$ if $\mathrm{v}_{\mathrm{j}} \in \mathrm{N}_{\mathrm{i}}$, and $\mathrm{a}_{\mathrm{ij}}=0$ otherwise A sequence edges is called a directed path, if the tail of the latter edge is the same as the head of previous pair, such as $\left(v_{i}, v_{i_{1}}\right),\left(v_{i_{1}}, v_{i_{3}}\right),\left(v_{i_{i}}, v_{i_{4}}\right), \ldots$

The Lapliacan matrix $\Sigma$ of a graph is defined as $L=\left[l_{i j}\right]_{m \times n}$, where $l_{i=}=\sum_{j=1}^{n} a_{i}$ and $l_{i j}=-a_{i j}$ if $a \neq j$. Obviously, a Laplacian matrix will be a nonnegative definite matrix.

\section{$\mathrm{H}_{2}$ and $\mathrm{H}_{\infty}$ Theory}

Consider the strictly proper system $T_{z w}$ which yields to the following dynamics:

$$
\begin{aligned}
& \dot{x}=A x+B w \\
& Z=C x
\end{aligned}
$$

where $A$ is a stable matrix.

\section{Lemma 1}

(Y. Jia [19]) The $\mathrm{L}_{2}$ norm of strict proper system (1) satisfies $\left\|T_{z w}\right\|_{2}^{2}=\operatorname{trace}\left(B^{T} L_{0} B\right)$, where Lo is the observability Gramian that satisfies

$$
A^{T} L_{0}+L_{0} A+C^{T} C=0
$$

\section{Lemma 2}

(Y. Jia [19]) Consider the system (1), the necessary and sufficient condition for $\left\|T_{z w}\right\|_{\infty}<\Upsilon$ is that the following Riccati equation has a positive definite solution

$$
A^{T} P+P A+\Upsilon^{-2} P B B^{T} P+C^{T} C<0
$$

\section{Linear algebra}

\section{Lemma 3}

(P. Lin et al. [5]) For matrix

$$
C=\left[\begin{array}{cccc}
\frac{n-1}{n} & -\frac{1}{n} & -\frac{1}{n} & \cdots \\
-\frac{1}{n} & \frac{n-1}{n} & -\frac{1}{n} & \cdots \\
\vdots & \ddots & \ddots & \ddots \\
-\frac{1}{n} & \cdots & -\frac{1}{n} & \frac{n-1}{n}
\end{array}\right]_{n \times n}
$$

and an arbitrary $n$-order Laplacian matrix $L$, there exists an orthogonal matrix $U=\left[U_{1}, \bar{U}_{1}\right]$, where $\bar{U}_{1}=[1 / \sqrt{n}, \ldots, 1 / \sqrt{n}]^{T}$, such that $U^{T} C U=\left[\begin{array}{cc}I_{n-1} & 0 \\ 0 & 0\end{array}\right]$ and $U^{T} L U=\left[\begin{array}{cc}U_{1}^{T} L U_{1} & 0 \\ \bar{U}_{1}^{T} L U_{1} & 0\end{array}\right]$.

\section{Lemma 4}

(CE. De Souza et al. [28]) Assume that D and E are real matrices with compatible dimensions. For an arbitrary scalar $\varepsilon$, we can get

$$
D E+E^{T} D^{T} \leq \epsilon^{-1} D D^{T}+\in E^{T} E
$$

\section{Lemma 5}

(Schur complement) Given a symmetric matrix $S \in \mathrm{R}^{\mathrm{n} \times \mathrm{n}}$ decomposed as

$$
S=\left[\begin{array}{ll}
S_{11} & S_{12} \\
S_{21} & S_{22}
\end{array}\right]
$$

where $\mathrm{S}_{11} \in \mathrm{R}^{\mathrm{n} \times \mathrm{n}}, \mathrm{S}_{12} \in \mathrm{R}^{(\mathrm{n}-\mathrm{r}) \times \mathrm{n}}$ and $\mathrm{S}_{22} \in \mathrm{R}^{(\mathrm{n}-\mathrm{r}) \times(\mathrm{n}-\mathrm{r})}$. Then $\mathrm{S}<0$ if and only if $\mathrm{S}_{11}<0$ and $S_{22}-S_{12}^{T} S_{11}^{-1} S_{12}<0$, or equivalently $\mathrm{S}_{22}<0$ and $S_{11}-S_{12} S_{22}^{-1} S_{12}^{T}<0$

\section{Problem Formulation}

In $[26,32]$, multi-UAVs (unmanned aerial vehicles) and satellite formations were supposed to disturbed by zero-mean Gaussian white noise. However, mathematically saying, the statistical characteristics of stochastic process may vary according to the information we have. In other words, the zero mean condition can not be guaranteed all the time. So we consider the singleintegrator multi-agent systems with identical dynamics, which are driven by energy bounded disturbances and white Gaussian noises.

$$
\dot{x}_{i}(t)=u_{i}(t)+w_{\infty i}(t)+b_{i} w_{0 i}(t)
$$

where $x_{i}(t)$ is the state of the $i$ th agent, $\mathrm{w}_{\infty \mathrm{i}}(\mathrm{t}) \in \mathrm{L}_{2}[0, \infty)$ is the energy bounded disturbance, and $\mathrm{w}(\mathrm{t})$ is the standard white Gaussian 
Citation: Ao Y, Jia Y (2018) Distributed $\mathrm{H}_{2} / \mathrm{H}_{\infty}$ Consensus Control for Multi-agent Systems with Directed Graph and Actuator Uncertainty. Int J Comput Softw Eng 3: 133. doi: https://doi.org/10.15344/2456-4451/2018/133

Page 3 of 9

We propose the following protocol based on the states of neighbors to achieve consensus. However, multi-agent systems are always in the face of calculation errors and variations of communication circumstances. Different from other consensus scheme, the communication perturbation is reflected on the multiplicative uncertainty of weight elements, so $\mathrm{H}_{\infty}$ theory can be utilized.

$$
u_{i}(t)=-\sum_{j \in N_{i}}\left[a_{i j}\left(1+\psi_{i j}(t)\right)\right]\left[x_{j}(t)-x_{i}(t)\right]
$$

where $a_{i j}$ is the parameter to be determined and $\psi_{i j}(t)$ is unknown multiplicative uncertainty of $a_{i j}$ with

$$
\psi_{i j}(t)=\left\{\begin{array}{cc}
\leq \bar{\psi}_{i j} & \text { if } i \neq j \text { and } a_{i j} \neq 0 \\
0 & \text { otherwise, }
\end{array}\right.
$$

In our work, the communication topology of the network is fixed and known. For example, it is like the layout of submarine optical cables and sensor networks, or some social relationship, in which the interaction topologies will not be changed over a period of time. Our objective is to find an optimal protocol to make the closed-loop system possess a desired level of disturbance rejection. For the $\mathrm{H}_{\infty}$ performance, on the one hand, the controlled output $z_{\infty i}(t)$ is present to value the difference between its state and the average state of all agents.

$$
z_{\infty i}(t)=x_{i}(t)-\frac{1}{n} \sum_{j=1}^{n} x_{j}(t)
$$

On the other hand, we expect that there is a tradeoff between the better system performance and the lower energy consumption. To consider the $\mathrm{H}_{2}$ performance of the multi-agent system, a novel controlled output is given.

$$
z_{0 i}(t)=\sum_{j=1}^{n} q_{i j}\left(x_{i}(t)-x_{j}(t)\right)+r_{i} u_{i}(t)
$$

where $q_{i j}>0$ corresponding to an undirected complete graph and $\mathrm{r}_{\mathrm{i}}>0$ are $\mathrm{H}_{2}$ performance coefficients. On the one hand, denoting $u_{t}(t)=R^{-1} 1_{n} 1_{n}^{T} x(t)+v(t)+\left\|T_{z_{o} w_{0}}\right\|_{2}^{2}=\int_{0}^{\infty}\left[x^{T}(t) Q^{2} x(t)+v^{T}(t) R^{2} v(t)\right] d t^{\prime}$ which is the interaction free cost function of LQR-based optimization problem of linear-consensus in $[17,18]$, where $Q=\left[q_{i j}\right] n \times n$ and $R=$ $\operatorname{diag}\left\{r_{1}, \ldots, r_{n}\right\}$. On the another hand, when $r_{i}=0$ and $\mathrm{Q}=\mathrm{C}, z_{\mathrm{O}_{i}}$ can be used to measure the static output variance of consensus under Guassian white noises. While $\left\|T_{z_{\infty} w_{\infty}}\right\|_{\infty}$ is adopted to guarantee the energy amplification from the expectation of the noises to the expectation of consensus output.

By utilizing protocol (7), the closed-loop dynamics of whole multiagent system can be written as

$$
\begin{aligned}
& \dot{x}(t)=-(L+\Delta L) x(t)+w_{\infty}(t)+B_{w_{0}}(t) \\
& z_{\infty}(t)=C_{x}(t) \\
& z_{0}(t)=(Q-R L) x(t)
\end{aligned}
$$

where $B=\operatorname{diag}\left\{b_{1}, \ldots, b_{n}\right\}$, and $C$ is defined in equation (4).

\section{Problem 1}

Given an $\mathrm{H}_{\infty}$ level $\gamma$, determine a Laplacian matrix $\mathrm{L}$ of the fixed interconnection of the multi-agent system (6) with multiplicative weight uncertainty satisfying (8), such that

$$
J=\inf _{L \in L_{n}} \sup _{\left|\psi_{i j}(t) \leq \bar{\psi}_{i j}\right|}\left\|T_{z_{0} w_{0}}\right\|_{2}
$$

under

1. the closed-loop multi-agent system (11) achieves asymptotically consensus for arbitrary $\psi_{i j}(t)$ satisfied (8) with the absence of $w_{\infty}(t)$ and $w_{0}(t)$ and the arbitrary initial states $x(0)$.

2. $\left\|T_{z_{\infty} w_{\infty}}\right\|_{\infty}<\Upsilon$ i.e $\int_{0}^{\infty} z_{\infty}^{T} z_{\infty}-\Upsilon^{2} w_{\infty}^{T} w_{\infty} d t<0$ for $\mathrm{w}_{0}(\mathrm{t})=0$, initial state $\mathrm{x}(0)=0$ and arbitrary $\mathrm{w}_{\infty} \in \mathrm{L}_{2}[0, \infty)$ and $\psi_{\mathrm{ij}}(\mathrm{t})$ satisfied (8).

\section{Lemma 6}

(W. Ren and RW. Beard [4]) The Laplacian matrix L of a directed graph $\mathrm{G}$ has a single zero eigenvalue (with eigenvector $1_{\mathrm{n}}$ ) if and only if the graph $\mathrm{G}$ contains a spanning tree. Moreover, other eigenvalues of $\mathrm{L}$ are strictly positive in this case.

In order to satisfy the first condition of Problem 1, it is a necessary condition in our work that the directed network of the multi-agent system contains a spanning tree.

\section{Lemma 7}

(P. Lin et al. [5]) Consider a directed graph G. Let $\mathrm{E}=\left[\mathrm{e}_{\mathrm{ij}}\right]$ and $\mathrm{F}$ $=\left[f_{i j}\right]$ be the 01-matrix. The rows and columns of $\mathrm{E}$ are indexed by the nodes and edges, while the columns and rows of $\mathrm{F}$ are indexed by the nodes and edges. $e_{i j}=1$ if the vertex $i$ is the tail of the edge $j, e_{i j}=$ 0 otherwise. And $\mathrm{f}_{\mathrm{ij}}=1$ if the vertex $\mathrm{j}$ is the head of the edge $\mathrm{i}, \mathrm{f}_{\mathrm{ij}}=0$ otherwise. Let weight matrix $\mathrm{W}=\operatorname{diag}\left\{\mathrm{w}_{1}, \mathrm{w}_{2}, \ldots, \mathrm{w}_{|\varepsilon|}\right\}$, where $\mathrm{w}_{\mathrm{p}}(\mathrm{p}$ $=1, \ldots,|\varepsilon|)$ is the weight of the pth edge of $G$ and $|\varepsilon|$ is the number of the edges. Then the relationship of the Laplacian matrix can be obtained $\mathrm{L}=\mathrm{EWD}$, where $\mathrm{D}=\mathrm{E}^{\mathrm{T}}-\mathrm{F}$.

\section{Main Results}

In this section, some main results of this paper are presented.

\section{Model Transformation}

Because of the singularity of the system matrix $-(L+\Delta L)$, which leads to $H_{2} / H_{\infty}$ theory invalid, some model transformations need to be conducted.

$$
\begin{aligned}
& \hat{x}(t)=x(t)-\frac{1_{n}}{n} \sum_{i=1}^{n} \int_{0}^{t}\left[w_{\infty}(s)+B_{w_{0}}(s)\right], \\
& \delta(t)=U_{1}^{T} \hat{x}(t) \text { and } \bar{\delta}(t)=\overline{\mathrm{U}}_{1}^{T} \hat{x}(t)
\end{aligned}
$$

\section{Remark 1}

Although $w_{\infty}(t)$ is a deterministic function with bounded norm while $w_{0}(t)$ is a random signal, sharing a common integral operator $\int_{0}^{\infty}$ will not create any confusions.

Then we can obtain that

$$
\begin{aligned}
& {\left[\begin{array}{c}
\dot{\delta}(t) \\
\dot{\bar{\delta}}(t)
\end{array}\right]=-\left[\begin{array}{c}
U_{1}^{T}(L+\Delta L) U_{1} 0 \\
U_{2}^{T}(L+\Delta L) U_{1} 0
\end{array}\right]\left[\begin{array}{l}
\delta(t) \\
\bar{\delta}(t)
\end{array}\right]+\left[\begin{array}{c}
U_{1}^{T} w_{\infty} \\
0
\end{array}\right]+\left[\begin{array}{c}
U_{1}^{T} w_{0} \\
0
\end{array}\right]} \\
& z_{\infty}=\left[\begin{array}{ll}
U_{1} & 0
\end{array}\right]\left[\begin{array}{c}
\delta(t) \\
\bar{\delta}
\end{array}\right] \\
& z_{0}=\left[Q U_{1}-R L U_{1} 0\right]\left[\begin{array}{l}
\delta(t) \\
\bar{\delta}(t)
\end{array}\right]
\end{aligned}
$$


Citation: Ao Y, Jia Y (2018) Distributed $\mathrm{H}_{2} / \mathrm{H}_{\infty}$ Consensus Control for Multi-agent Systems with Directed Graph and Actuator Uncertainty. Int J Comput Softw Eng 3: 133. doi: https://doi.org/10.15344/2456-4451/2018/133

Page 4 of 9

Consider the $\mathrm{H}_{2}$ and $\mathrm{H}_{\infty}$ performance $\left\|T_{z_{0} w_{0}}\right\|_{2}$ and $\left\|T_{z_{\infty} w_{\infty}}\right\|_{\infty}$ of the multi-agent system, by Lemma 7 , we study the reduced dimension system

$$
\begin{aligned}
T_{z w}^{f}(s) & =\left[\begin{array}{ccc}
-U_{1}^{T} E W D U_{1}-U_{1}^{T} E \Delta W(t) D U_{1} & U_{1}^{T} & U_{1}^{T} B \\
U_{1} & 0 & 0 \\
Q U_{1}-R E W D U_{1} & 0 & 0
\end{array}\right] \\
& =\left[\begin{array}{ccc}
A_{f}+\Delta A_{f}(t) & B_{\infty f} & B_{0 f} \\
C_{\infty f} & 0 & 0 \\
C_{o f} & 0 & 0
\end{array}\right]
\end{aligned}
$$

\section{Lemma 8}

The following conditions are equivalent:

1. The protocol (7) can solve the Problem 1.

2. For a multi-agent system with digraph which contains a spanning tree, there exist symmetric positive definite matrices $\tilde{X}_{2}, X_{\infty}$ and a $|\varepsilon|$-dimensional diagonal positive definite matrix $\mathrm{W}$ solving the following non-convex optimization problem:

\section{Problem 2}

$$
\begin{aligned}
& J=\min _{X_{\infty}, \tilde{X}_{2} \text { and } L \in L_{n}} \sup _{\psi_{i}(t)} \operatorname{trace}\left(B_{o f}^{T} \tilde{X}_{2} B_{0 f}\right) \\
& A_{f}^{T} \tilde{X}_{2}+\tilde{X}_{2} A_{f}+C_{f}^{T} C_{f}=0 \\
& {\left[\begin{array}{c}
\left.\left(A_{f}+\Delta A_{f}\right)^{T} X_{\infty}+X_{\infty}\left(A_{f}+\Delta A_{f}\right)+C_{f}^{T} C_{f} X_{\infty} B_{\infty f}\right]<0 \\
B_{\infty f}^{T} X_{\infty}-\Upsilon^{2} I
\end{array}\right]}
\end{aligned}
$$

And the cost function $\mathrm{J}$ is the Laplacian matrix $\mathrm{L}$.

Proof Equation (16) can be obtained readily by the definition of $L_{2}$ norm in Lemma 1 . And the $H_{\infty}$ constraint can be transformed into inequlity (17) referred to [19].

\section{Conditions for $\mathrm{H}_{2} / \mathrm{H}_{\infty}$ Consensus}

\section{Lemma 9}

(Elimination lemma) $P=[\bar{P},-I], H, \mathrm{H}$ are given matrices with compatible dimensions, and $\mathrm{H}$ is a symmetric matrix. Denote $N_{P}=\left[I, \bar{P}^{T}\right]^{T}$.

Then, there exists a matrix $X=\left[X_{1}^{T}, X_{2}\right]^{T}$ where $\mathrm{X}_{2}$ is a positive definite diagonal matrix, such that

$$
H+P^{T} X^{T}+X P<0
$$

if and only if

$$
N_{P}^{T} H N_{P}<0
$$

Proof Necessity can be obtained noticeably. Next, we use a constructive approach to prove the sufficiency. A full column rank matrix $\bar{V}=[0, I]^{T}$ is introduced to expand NP into a group of basis of the entire space, i.e. $V=\left[N_{P}, \bar{V}\right]$ is an invertible square matrix. So inequality (18) holds if and only if there exists X such that

$$
V^{T}=\left(H+P^{T} X^{T}+X P\right) V<0
$$

holds. According to the block of $V$, we assume that

$$
V^{T} H V=\left[\begin{array}{ll}
H_{11} & H_{12} \\
H_{12}^{T} & H_{22}
\end{array}\right]
$$

and obtain that

$$
V^{T} X P V=\left[\begin{array}{cc}
0-X_{1} & -\bar{P}^{T} X_{2} \\
0 & -X_{2}
\end{array}\right]
$$

Substituting equations (21) and (22) into inequation (20) so that it can be rewritten as

$$
\left[\begin{array}{cc}
H_{11} & H_{12}-X_{1}-\bar{P}^{T} X_{2} \\
H_{12}^{T}-X_{1}^{T}-X_{2} \bar{P} & H_{22}-X_{2}-X_{2}
\end{array}\right]<0
$$

By Schur complement lemma, inequation (23) holds if and only if $H_{11}<0$ and

$$
H_{22}-X_{2}-X_{2}-\left(H_{12}^{T}-X_{1}^{T}-X_{2} \bar{P}\right) H_{11}^{-1}\left(H_{12}-X_{1}-\bar{P}^{T} X_{2}\right)<0
$$

$H_{11}<0$ is obtained by condition (19) and it is obvious that for matrix $\mathrm{X}_{1}=-\bar{P}^{T} X_{2}$, there exists a positive diagonal matrix $\mathrm{X} 2$ such that inequality (24) holds. So the sufficiency is proofed.

To simplify the conditions of optimization Problem 2, the following notations are stated:

$$
\begin{aligned}
N\left(X_{2}\right) & =\left[\begin{array}{ccc}
U_{1}^{T} Q^{2} U_{1} & -X_{2} U_{1}^{T} E-U_{1}^{T} Q R E \\
-E^{T} U_{1} X_{2}-E^{T} R Q U_{1} & E^{T} R^{2} E
\end{array}\right] \\
L\left(X_{\infty}\right) & =\left[\begin{array}{cccc}
\mu U_{1}^{T} E_{2}^{T} E_{2} U_{1}+I & X_{\infty} & 0 & -X_{\infty} U_{1}^{T} E_{1} \\
X_{\infty} & -\Upsilon^{2} I & 0 & 0 \\
0 & 0 & -\mu \psi^{-2} & 0 \\
-E_{1}^{T} U_{1} X_{\infty} & 0 & 0 & 0
\end{array}\right]
\end{aligned}
$$

\section{Theorem 1}

Assume the directed graph of multi-agent system (6) contains a spanning tree, distribute $\mathrm{H}_{2} / \mathrm{H}_{\infty}$ synthesis problem can be transformed to the following non-convex constraint optimization problem:

\section{Problem 3}

If there exist symmetric positive definite matrices $X_{\infty}, X_{2}$, matrices $K_{21}, K_{\infty 1}, K_{\infty 2,}, K_{\infty 3}$ diagonal matrices $Y, Z, F_{2}, F_{\infty}$ and a positive scalar $\mu$, such that

$$
\hat{J}=\min _{\substack{\mu, X_{2}, X_{\infty}, K_{21}, K_{\infty 1}, K_{\infty 2,}, K_{\infty 3} \text { and } Y, Z, F_{2}, F_{\infty} \in D_{\epsilon \mid}}} \operatorname{trace}\left(B^{T} U_{1} X_{2} U_{1}^{T} B\right)
$$

under

$$
\begin{aligned}
& Z-F_{\infty} F_{2}^{-1} Y=0 \\
& N\left(X_{2}\right)+\operatorname{sym}\left(\left[\begin{array}{c}
K_{2}^{T} \\
-I
\end{array}\right]\left[Y D U_{1} F_{2}\right]\right)<0 \\
& L\left(X_{\infty}\right)+\operatorname{sym}\left(\left[\begin{array}{c}
K_{\infty 1}^{T} \\
K_{\infty 2}^{T} \\
K_{\infty 3}^{T} \\
-I
\end{array}\right]\left[Z D U_{1}-Z F_{\infty}\right]\right)<0
\end{aligned}
$$


Citation: Ao Y, Jia Y (2018) Distributed $\mathrm{H}_{2} / \mathrm{H}_{\infty}$ Consensus Control for Multi-agent Systems with Directed Graph and Actuator Uncertainty. Int J Comput Softw Eng 3: 133. doi: https://doi.org/10.15344/2456-4451/2018/133

Page 5 of 9

It provides a sub-optimal solution to the $H_{2} / H_{\infty}$ consensus problem and the upper bound $\hat{J}$ of the $L_{2}$ norm of the multi-agent system, by solving Problem 3.

At the optimum, the sub-optimal weight matrix can be given by

$$
W^{*}=-F_{\infty}^{*-1} Z^{*}=-F_{2}^{*-1} Y^{*}
$$

Proof By Lemma 4, the following inequality can be obtained for an arbitrary scalar $\mu>0$

$$
\Delta A_{f}(t)^{T} X_{\infty}+X_{\infty} \Delta A_{f}(t) \leq \mu U_{1}^{T} D^{T} D U_{1}+\frac{1}{\mu} X_{\infty} U_{1}^{T} E W \bar{\psi}^{2} W E^{T} U_{1} X_{\infty}
$$

By Lemma 5, the $\mathrm{H}_{\infty}$ condition $\left\|T_{z_{\infty} w_{\infty}}\right\|_{\infty}<\Upsilon$ holds, if there exists a positive definite matrix $\mathrm{X}_{\infty}$ such that

$$
\left[\begin{array}{ccc}
-A_{f}^{T} X_{\infty}-X_{\infty} A_{f}+I_{n-1}+\mu U_{1}^{T} D^{T} D U_{1} & X_{\infty} & X_{\infty} U_{1}^{T} D W \\
X_{\infty} & -\Upsilon I & 0 \\
W E^{T} U_{1} X_{\infty} & 0 & -\mu \psi^{-2}
\end{array}\right]<0
$$

Substitute $L\left(X_{\infty}\right)$ and $N\left(X_{2}\right)$ into the constraint (16) and (17). Then the $\mathrm{H}_{2} / \mathrm{H}_{\infty}$ synthesis problem can be rewritten as

\section{Problem 4} under

$$
\min _{\mu, X_{2}, X_{\infty}, \text { and } W \in D_{\in \mid}} \operatorname{trace}\left(B^{T} U_{1} X_{2} U_{1}^{T} B\right)
$$

$$
\begin{aligned}
& {\left[I U_{1}^{T} D^{T} W\right] N\left(X_{2}\right)\left[\begin{array}{c}
I \\
W D U_{1}
\end{array}\right]<0} \\
& {\left[\begin{array}{cccc}
I & 0 & 0 & U_{1}^{T} D^{T} W \\
0 & I & 0 & 0 \\
0 & 0 & I & -W
\end{array}\right] L\left(X_{\infty}\right)\left[\begin{array}{ccc}
I & 0 & 0 \\
0 & I & 0 \\
0 & 0 & I \\
W D U_{1} & 0 & -W
\end{array}\right]<0}
\end{aligned}
$$

It indicates that the feasible region of $\mathrm{W}$ of Problem 4 is contained in the feasible region of Problem 2 by inequality (29). And we can readily get that $X_{2} \geq \tilde{X}_{2}$ where $\mathrm{X}_{2}$ is governed by inequality (31) while $\tilde{X}_{2}$ yields to equation (16). So it gives a upper bound of the $\mathrm{L}_{2}$ norm of the multi-agent system by solving Problem 4 .

Using Lemma 9 to slack some slack variables, we can obtain that Problem 4 equals to

$$
\min _{\substack{\mu, X_{2}, X_{\infty}, F_{21}, F_{\infty 1}, F_{\infty 2}, F_{\infty 3}, \text { and } W, F_{22}, F_{\infty 4} \in D_{\in \mid}}} \operatorname{trace}\left(B^{T} U_{1} X_{2} U_{1}^{T} B\right)
$$

under

$$
\begin{aligned}
& N\left(X_{2}\right)+\operatorname{sym}\left(\left[\begin{array}{l}
F_{21}^{T} \\
F_{22}
\end{array}\right]\left[W D U_{1}-I\right]\right)<0 \\
& L\left(X_{\infty}\right)+\operatorname{sym}\left(\left[\begin{array}{c}
F_{\infty}^{T} \\
F_{\infty 2}^{T} \\
F_{\infty 3}^{T} \\
F_{\infty 4}^{T}
\end{array}\right]\left[W D U_{1} 0-W-I\right]\right)<0
\end{aligned}
$$

where $\mathrm{F}_{22}, \mathrm{~F}_{\infty 4} \in \mathrm{D}_{|\varepsilon|}$ are positive definite diagonal matrices and other slack variables are real matrix with compatible dimensions. State that

$$
\begin{aligned}
& Y=-F_{22} W \mathrm{Z}=-F_{\infty 4} W K_{2}=-F_{22}^{-T} F_{21} \\
& K_{\infty 1}=-F_{\infty 4}^{-T} F_{\infty 1} K_{\infty 2}=-F_{\infty 4}^{-T} F_{\infty 2} \\
& K_{\infty 3}=-F_{\infty 4}^{-T} F_{\infty 3} \text { and } F_{2}=F_{22} F_{\infty}=F_{\infty 4}
\end{aligned}
$$

Since matrices $Y, Z, F_{2}$ and $F_{\infty}$ are diagonal matrices with equality relationship (26), Problem 3 is obtained and Theorem 1 is proofed.

\section{Remark 2}

However, because of the nonlinear equational constraint (26), it is hard to utilize a numerical procedure to solve the constrained optimization Problem 3. We relax it by assuming that

$$
F_{\infty}=F_{2}=F \text { and } Y=Z
$$

Then we solve the following BMI constrained optimization problem

\section{Problem 5}

If there exist symmetric positive definite matrices $\mathrm{X}_{\infty}, \mathrm{X}_{2}$, matrices $\mathrm{K}_{2}, \mathrm{~K}_{\infty 1}, \mathrm{~K}_{\infty 2}, \mathrm{~K}_{\infty 3}$, diagonal matrices $\mathrm{Z}, \mathrm{F}$ and a positive scalar $\mu$ solution of the optimization problem:

$$
\min _{\substack{\mu, X_{2}, X_{\infty}, K_{2}, K_{\infty 1}, K_{\infty 2}, K_{\infty 3}, \text { and } Z, F \in D_{|\in|}}} \operatorname{trace}\left(B^{T} U_{1} X_{2} U_{1}^{T} B\right)
$$
under

$$
\begin{aligned}
& N\left(X_{2}\right)+\operatorname{sym}\left(\left[\begin{array}{c}
K_{2}^{T} \\
-I
\end{array}\right]\left[Z D U_{1} F\right]\right)<0 \\
& L\left(X_{\infty}\right)+\operatorname{sym}\left(\left[\begin{array}{c}
K_{\infty 1}^{T} \\
K_{\infty 2}^{T} \\
K_{\infty 3}^{T} \\
-I
\end{array}\right]\left[Z D U_{1} 0-Z F\right]\right)<0
\end{aligned}
$$

to get an upper bound on the $\mathrm{L}_{2}$ norm $\left\|T_{z_{0} w_{0}}\right\|_{2}$. And optimal weight matrix $\mathrm{W}^{*}=-\mathrm{F}^{*-1} \mathrm{Z}^{*}$.

A coordinate-descent based iterative procedure similar to [15] is used to solve the BML optimal problem.

\section{Numerical Algorithm Description}

\section{Algorithm 1}

1. Step 1: For a directed graph $\mathrm{G}$ which contains a spanning tree, choose the initial matrices $\mathrm{K}_{2}=\mathrm{K}_{\infty 1}=\mathrm{W}_{0} \mathrm{DU}_{1}$ and $\mathrm{K}_{\infty 2}=\mathrm{K}_{\infty 3}=0$, where $\mathrm{W}_{0}$ is an appropriate matrix with all eigenvalues positive.

2. Step 2k: Pick the optimal solution matrices $\mathrm{K}_{2}, \mathrm{~K}_{\infty 1}, \mathrm{~K}_{\infty 2}$ corresponding to the minimum situation in the last step. Then solve the optimization linear matrix inequality (LMI) problem $\beta_{2 k}^{2}=\min _{\mu, X_{2}, X_{\infty}, \text { and } Z, F \in D_{|\in|}} \operatorname{trace}\left(B^{T} U_{1} X_{2} U_{1}^{T} B\right)$ under inequality constraint (36)

3. Step $2 \mathrm{k}+1$ : Pick the diagonal optimal solution matrices $Z$, $F$ corresponding to the minimum situation in the last step. Then solve the convex optimization LMI problem $\beta_{2 k+1}^{2}=\min _{\mu, X_{2}, X_{\infty}, K_{2}, K_{\infty 1, K_{\infty 2}, K_{\infty 3}}} \operatorname{trace}\left(B^{T} U_{1} X_{2} U_{1}^{T} B\right)$ under inequality constraint (36)

4. Final step: If $\beta_{2 \mathrm{k}}-\beta_{2 \mathrm{k}+1}<\varepsilon$, then stop. The sub-optimal weight matrix of the digraph of the first-order multi-agent system is $W_{\beta}^{*}=-F^{-1} Z$ obtained, and the upper bound on the $\mathrm{L}_{2}$ norm $\left\|T_{z_{0} w_{0}}\right\|_{2}$ is obtained as $\beta_{2 k+1}^{1 / 2}$; else go on to step $2 \mathrm{k}+2$. 
Citation: Ao Y, Jia Y (2018) Distributed $\mathrm{H}_{2} / \mathrm{H}_{\infty}$ Consensus Control for Multi-agent Systems with Directed Graph and Actuator Uncertainty. Int J Comput Softw Eng 3: 133. doi: https://doi.org/10.15344/2456-4451/2018/133

Page 6 of 9

\section{Remark 3}

From the description of the algorithm, it can be guaranteed that the target function will not increase at each iteration. It still will be a challenge task to proof the global convergency mathematically. However it has turned out effective in simulation and practice by choosing an appropriate initial value in the step 1 of Algorithm 1.

\section{Numerical simulations}

Consider a first-order multi-agent system with 5 agents. Their communication connection relationships are as Figure 1. Then we design a Laplacian matrix to solve the $H_{2} / H_{\infty}$ sub-optimization problem. In other words, the weight of each edge need to be determined. By using Remark 2 and Algorithm 1, setting $\gamma=1, \varepsilon=$ 0.01 and $\bar{\psi}_{i j}=0.05$ then we can obtained the $H_{2} / H_{\infty}$ sup-optimal solution $\mathrm{W} \in \mathrm{D}_{7}$ and $\mathrm{L} \in \mathrm{L}_{5}$, where $\mathrm{W}=\operatorname{diag}\{3.1043,1.0915,0.6263,0.1846$, $0.5366,1.2051,0.1403\}$ and the parameters of protocol (7) and the upper bound $\beta_{2 k+1}^{1 / 2}=2.4685$ are also reaped.

$$
L=\left[\begin{array}{ccccc}
0.6769 & 0 & 0 & -0.5366 & -0.1403 \\
-3.1043 & 3.1043 & 0 & 0 & 0 \\
0 & -1.0915 & 1.0915 & 0 & 0 \\
0 & -0.6263 & -0.1846 & 0.8108 & 0 \\
-1.2051 & 0 & 0 & 0 & 1.2051
\end{array}\right]
$$

It is revealed from Figure 2 that the 5 agents can achieve consensus with good performance against Guassian white noises. And $H_{\infty}$ performance is illustrated where $w_{\infty i}$ are sinusoidal disturbances in Figure 3. Figure 4 reveals the trajectory of the sum of the impulse response energy of $\mathrm{z}_{0}$, which is equivalent to the $L_{2}$ norm of the closedloop system $\quad \|, z_{0}$ and $n_{0} \mid$ it is found approaching the upper bound we obtai $\beta_{2 k+1}^{1} e_{k}^{d}$ hereinbefore.

Compared with [5], our work goes a step further, not only because of the design of the Laplacian matrix we proposed, but also the consideration of $\mathrm{H}_{2}$ performance. Using $\mathrm{H}_{\infty}$ approach in [5], assuming that the weight of each edge in graph 1 is equivalent, we can verify that the below equation; minimizes the $L_{2}$ norm of system (6) with $\left\|T_{z_{\infty} w_{\infty}}\right\|_{\infty}<1$. And in this case, the sum of the impulse response energy of $z_{0}$ is shown in Figure 5.

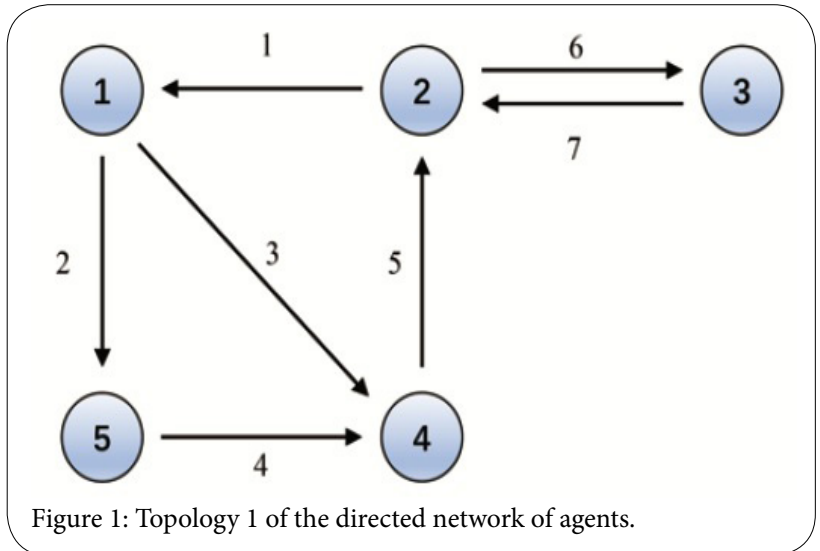

$$
L=\left[\begin{array}{ccccc}
2.762 & 0 & 0 & -1.381 & -1.381 \\
-1.381 & 1.381 & 0 & 0 & 0 \\
0 & -1.381 & -1.381 & 0 & 0 \\
0 & -1.381 & -1.381 & 2.762 & 0 \\
-1.381 & 0 & 0 & 0 & 1.381
\end{array}\right]
$$

Two extra examples are provided for comparison. The topologies of multiagent systems are shown in Figure 6 and Figure 7. $H_{2}$ performance results achieved by $H_{2} / H_{\infty}$ approach and $H_{\infty}$ approach are illustrated by Table. 1 , which indicates that $H_{2} / H_{\infty}$ approach can achieve a better robust $H_{2}$ performance compared with $H_{\infty}$ approach with $\left\|T_{z_{\infty} w_{\infty}}\right\|_{\infty}<1$.

\section{Conclusion}

This paper has studied the disturbance rejection problem of first-order multiagent system consensus with directed interaction topology. $L_{2}$ norm and $H_{\infty}$ norm were utilized to measure the system consensus performance. Under the premise of satisfying given $H_{\infty}$ performance, a new type of measure output contained more generality was proposed, and a relaxation approach with lower conservatism was presented to search the sub-optimal solution of the $H_{2} / H_{\infty}$. Notably, a novel elimination lemma was obtained. In future work, high-order multi-agent systems with parameter uncertain and time-delay will be

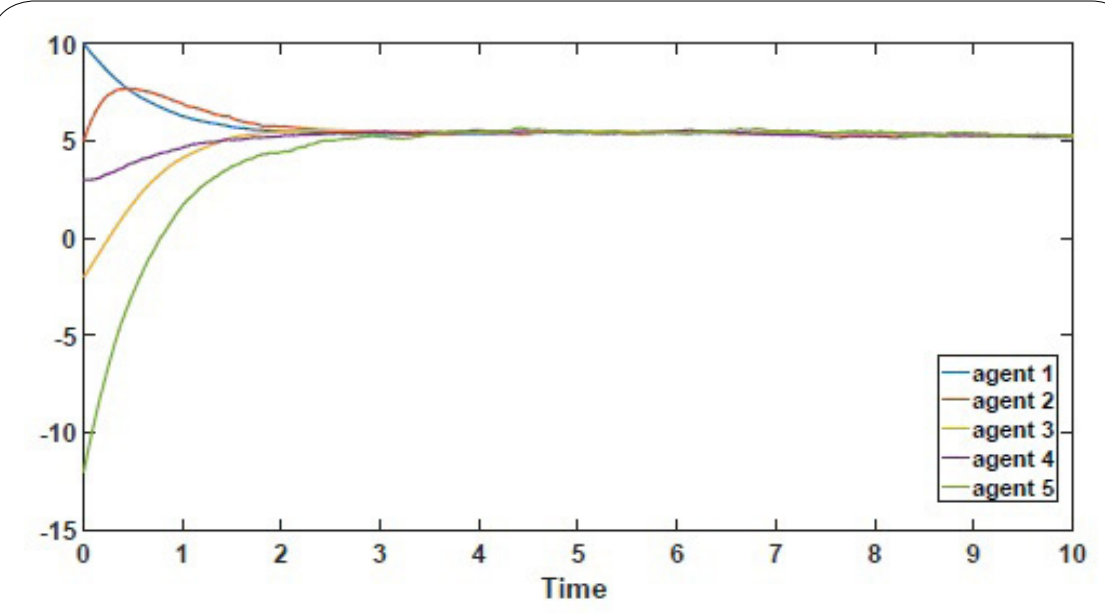

Figure 2: The state trajectories of the agents with Gaussian noises. 
Citation: Ao Y, Jia Y (2018) Distributed $H_{2} / H_{\infty}$ Consensus Control for Multi-agent Systems with Directed Graph and Actuator Uncertainty. Int J Comput Softw Eng 3: 133. doi: https://doi.org/10.15344/2456-4451/2018/133

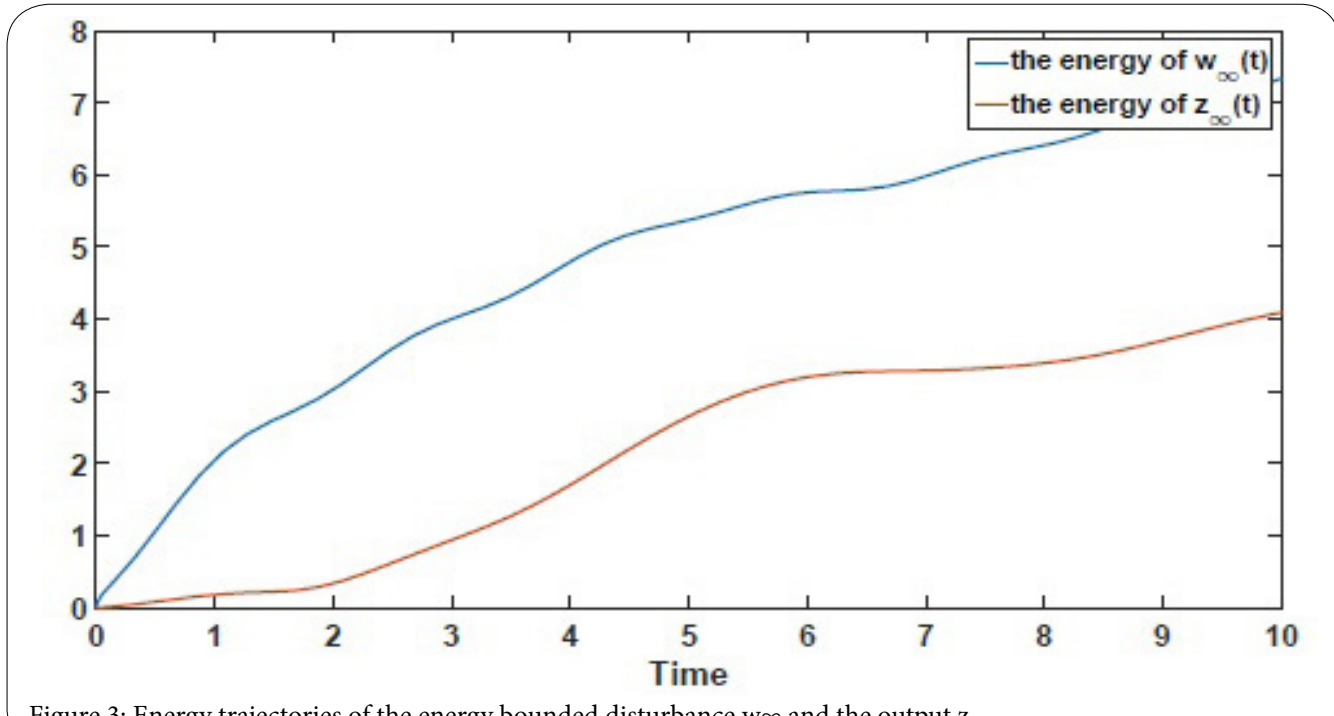

Figure 3: Energy trajectories of the energy bounded disturbance $w \infty$ and the output $z_{\infty}$
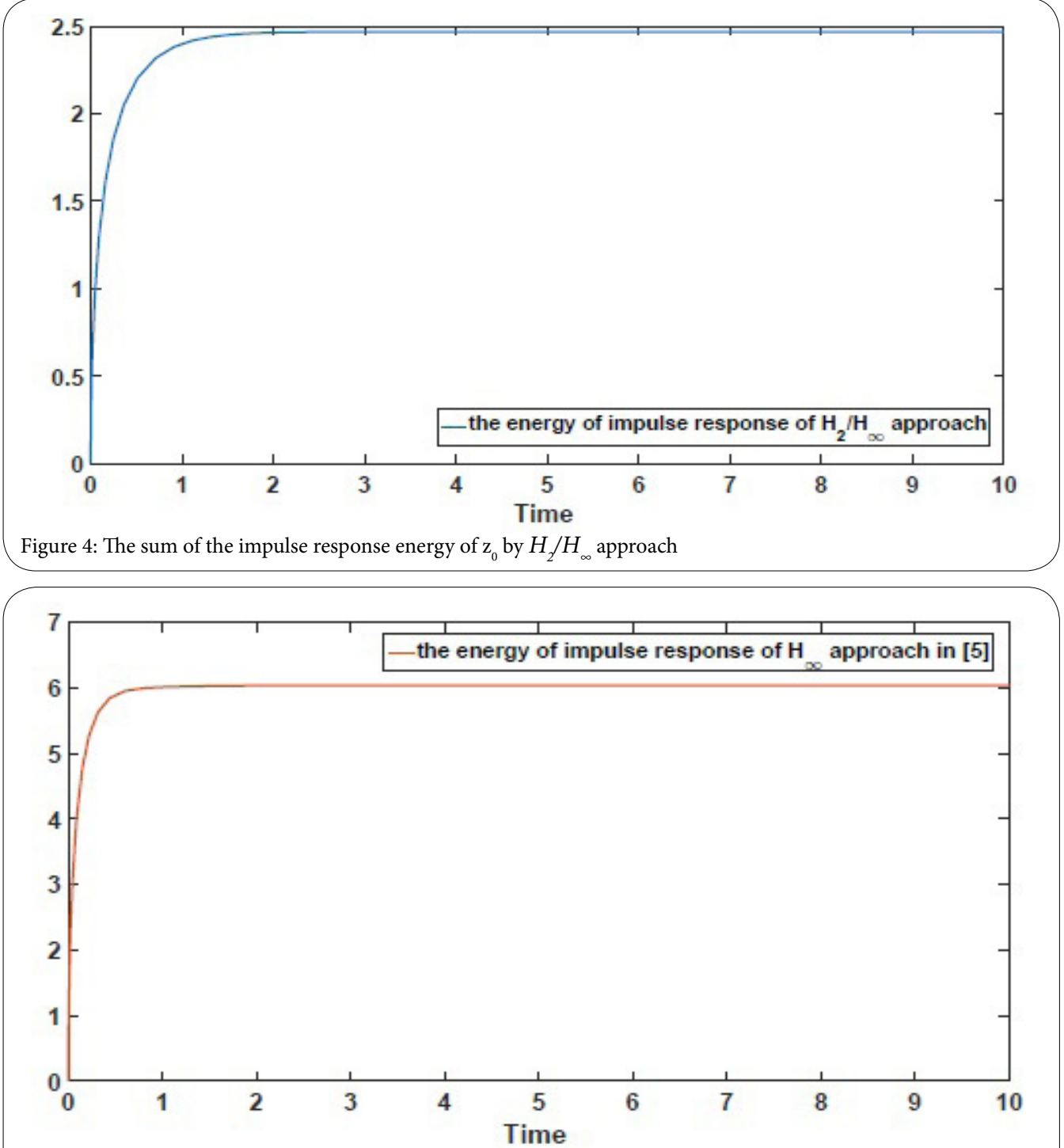

Figure 5: The sum of the impulse response energy of $\mathrm{z}_{0}$ by $\mathrm{H}_{\infty}$ approach in [5]. 
Citation: Ao Y, Jia Y (2018) Distributed $H_{2} / H_{\infty}$ Consensus Control for Multi-agent Systems with Directed Graph and Actuator Uncertainty. Int J Comput Softw Eng 3: 133. doi: https://doi.org/10.15344/2456-4451/2018/133

Page 8 of 9

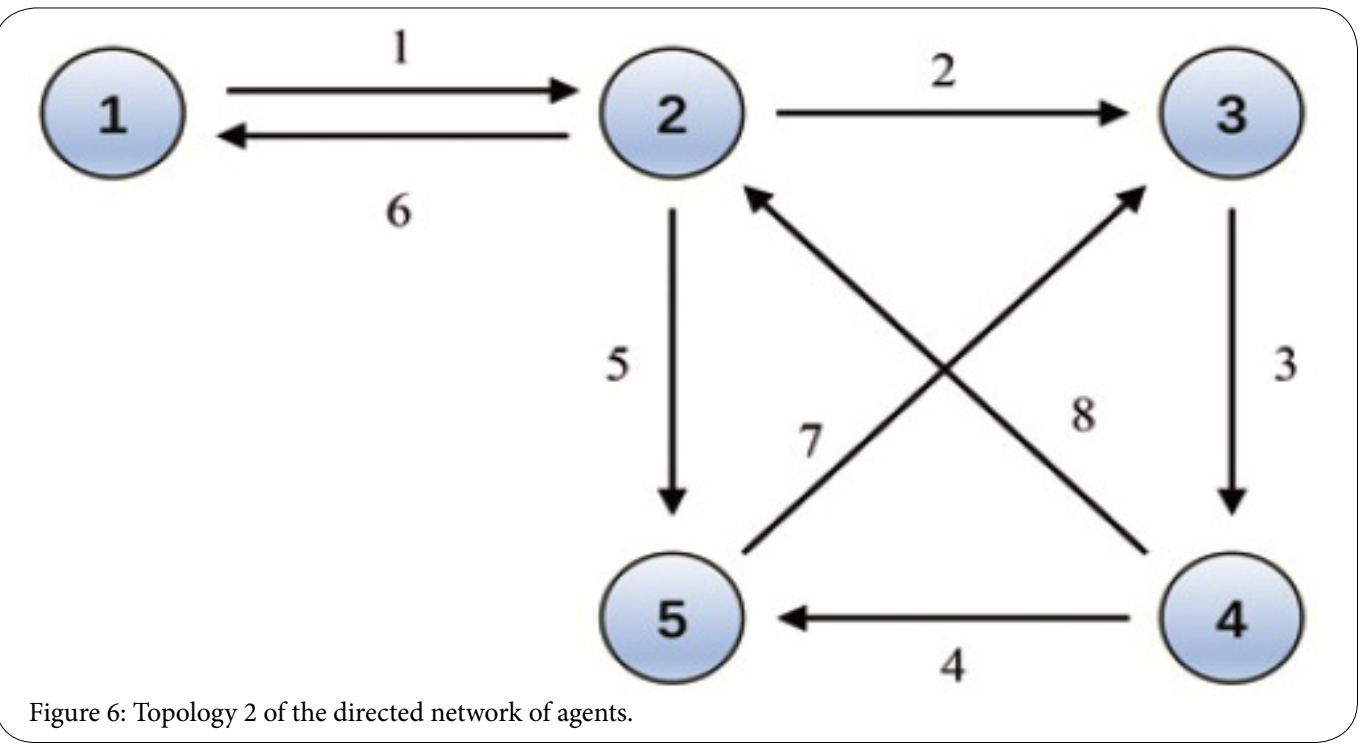

Figure 6: Topology 2 of the directed network of agents.
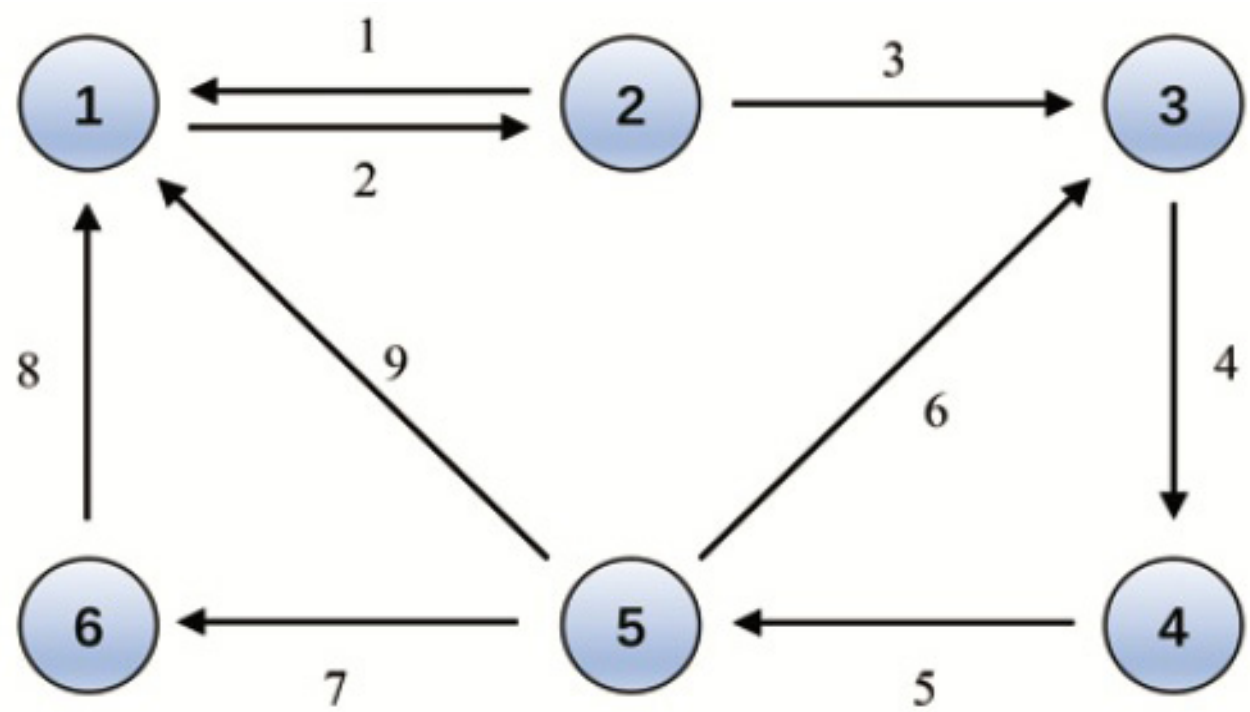

Figure 7: Topology 3 of the directed network of agents.

\begin{tabular}{|l|l|l|}
\hline Multi agent system of & Sub-optimal $\mathrm{L}_{2}$ norm by $H_{2} / H_{\infty}$ approach & Sub-optimal $\mathrm{L}_{2}$ norm by $H_{\infty}$ approach in $[5]$ \\
\hline Topology 1 & 2.4685 & 6.0638 \\
\hline Topology 2 & 2.7202 & 4.3505 \\
\hline Topology 3 & 3.7241 & 4.7494 \\
\hline Table $1:$ & & \\
\hline
\end{tabular}

considered. For these cases, measurement errors caused by noises and higher conservatism caused by the larger number of the agents will also be the challenges to overcome.

\section{Acknowledgment}

This work was supported by the NSFC (61520106010, 61327807, $61521091,61134005)$ and the National Basic Research Program of China (973 Program: 2012CB821200, 2012CB821201).

\section{Competing Interests}

The authors declare that no competing interests exist.

\section{References}

1. Jadbabaie A, Lin J, Morse S (2003) Coordination of groups of mobile autonomous agents using nearst neighbor rules. IEEE Trans Autom Control 48: 988.

2. Olfati-Saber R, Murray RM (2004) Consensus problems in networks of agents with switching topology and time-delays. IEEE Trans. Autom. Control 49: 1520

3. Ren W, Beard RW (2005) Consensus seeking in multiagent systems under dynamically changing interaction topiologies. IEEE Trans Autom Control 50: 655.

4. Ren W, Atkins E (2006) Distributed multi-vehicle coordinated control via local information exchange. Int J Robust Nonlin 17: 1002. 
Citation: Ao Y, Jia Y (2018) Distributed $H_{2} / H_{\infty}$ Consensus Control for Multi-agent Systems with Directed Graph and Actuator Uncertainty. Int J Comput Softw Eng 3: 133. doi: https://doi.org/10.15344/2456-4451/2018/133

Page 9 of 9

5. Lin P, Jia Y, Li L (2008) Distributed robust $H$ consensus control in directed networks of agents with time-delay. Syst Control Lett 57: 643.

6. Lin P, Jia $Y(2010)$ Robust $H_{\infty}$ consensus analysis of a class of secondorder multiagent systems with uncertainty. IET Control Theor Appl 4: 487.

7. Liu Y, Jia Y (2010) Consensus problem of high-order multi-agent systems with external disturbances: An $H_{\infty}$ analysis approach. Int. J. Robust Nonlin Control 20: 1579

8. Cui $Y$, Jia $Y(2014)$ Robust $L_{2}-L_{\infty}$ consensus control for uncertain high-order multi-agent systems with time delay. Int. J. Syst. Sci. 45: 427.

9. Li Z, Duan Z, Chen G (2011) On $H_{\infty}$ and $H_{2}$ performance regions of multiagent systems. Automatica 47: 797.

10. Zhao Y, Duan Z, Wen G, Chen G (2012) Distributed $H_{\infty}$ consensus of multi-agent systems: A performance region-based approach. Int J Control 85:332.

11. Xiao L, Boyd S, Kim S (2007) Distributed average consensus with leastmean-square deviation. Journal of Parall Distri Computing 67: 33-46.

12. Li T, Zhang J (2008) Mean Square Average Consensus of Multi-agent Systems with Time-varying Topologies and Stochastic Communication Noises. Proceedings of the 27th Chinese Control Conference, Kunming, China.

13. Gupta V, Hasssibi B, Murray M (2005) A Sub-optimal algorithm to synthesize control laws for a network of dynamic agents. Int J Control 78: 1302.

14. Bernstein DS, Haddad WM (1989) LQG control with an $H$ performance bound: Ariccati equation approach. IEEE Trans. Autom Control 34: 293.

15. Arzelier D, Peaucelle D (2002) An Iterative Method for $H_{2} / H_{\infty}$ Synthesis via Static Output-feedback. Proceeding of 41st IEEE Conference on Dcision and Control, Las Vegas, NV, Dec.

16. Khargonekar PP, Rotea MA (1991) Mixed $H_{2} / H_{\text {on }}$ control: A convex optimization approach. IEEE Trans. Autom. Control 36: 824.

17. Cao Y, Ren W (2010) Optimal linear-consensus algorithms LQR perspective. IEEE Trans Syst Man CySyst 40: 819.

18. Cao Y, Ren W (2009) LQR-based Optimal Linear Consensus Algorithms. American Control Conference, St Louis, MO, Jun.

19. Jia $Y(2007)$ Robust $H_{\infty}$ control. Science Press, China.

20. Cortes J, Bullo F (2003) Non smooth coordination and geometric optimization via distributed dynamical systems. SIAMJ Control Optim 51: 163-189.

21. Paganini F, Doyle J, Low S (2002) Scalable laws for stable network congestion control. IEEE Conference on Decision \& Control, Orlando.

22. Lynch NA (1997) Distributed Algorithms. San Mateo, CA: Morgan Kaufmann.

23. Yamaguchi $\mathrm{H}$ (1999) A cooperative hunting behavior by mobile robot troops, Proceedings of the 1998. IEEE International Conference on Robotics \& Automation 18: 3204-3209.

24. Schaub H, Vadali SR, Junkins JL, Alfriend KT (2000) Spacecraft formation ying control using mean orbital elements. J Astronaut Sci 48: 69-87.

25. Massioni P, Verhaegen M (2009) Distributed control for identical dynamically coupled systems: A decomposition approach. IEEE Trans on Autom Control 54: 124-135.

26. Mhadami R, Shafai B (2010) Distributed H2 control of multi-agent dynamic systems: Continuous-time case. American Control Conference.

27. Scherer C, Gahinet P, Chilali M (1997) Multiobjective output-feedback control via LMI optimization. IEEE Trans on Autom Control 42: 896-911.

28. Souza CE, Li X (1999) Delay-dependent robust $H_{\infty}$ control of uncertain linear state-delayed systems. Automatica 35: 1313-1321.

29. Wang J, Duan Z, Li Z, Wen G (2014) Distributed $H$ and $H_{2}$ consensus control in directed networks. IET Control Theor Appl 8: 193-201.

30. Xie CH, Yang G (2016) Cooperative guaranteed cost fault-tolerant control for multi-agent systems with time-varying actuator faults. Neuro computing 214: 382-390.
31. Young GF, Scardovi L, Leonard NE (2010) Robustness of Noisy Consensus Dynamics with Directed Communication. American Control Conference.

32. Valasek J, Gunnam K, Kimmett J, Tandale MD, Junkins JL, et al. (2005) Vision-based sensor and navigation system for automonous air refueling. $J$ Guid Contr Dynam 28: 979-989. 